\title{
Exercise generation with the system Passarola
}

\author{
José João Almeida ${ }^{1}$ Isabel Araújo ${ }^{2}$ Irene Brito $^{2}$ Nuno Carvalho ${ }^{1}$ \\ Gaspar J. Machado ${ }^{2}$ Rui M. S. Pereira ${ }^{2}$ Georgi Smirnov $^{3}$ \\ ${ }^{1}$ Centre of Informatics, University of Minho, Portugal \\ ${ }^{2}$ Centre of Mathematics, University of Minho, Portugal \\ ${ }^{3}$ Centre of Physics, University of Minho, Portugal
}

\begin{abstract}
A robust multi-domain coverage exercise generation system usually works with answers of simple types (e.g. multiplechoice, Boolean, integer, or file comparison). In this paper we describe Passarola, a simple, yet powerful, exercise generation system and its language that anyone with no computer science background can use to develop exercises. It may include a collection of heterogeneous objects allowing the usage of complex elements. Its main characteristics are the use of simple reusable templates, simple and rich types, and rich notation and syntax (LaTeX based) for questions, solutions, and answers.
\end{abstract}

Keywords: Passarola, exercise generation system, self-regulating study.

\section{Introduction}

When implementing the Bologna education reform in Portuguese universities, the number of contact hours of the courses decreased (considerably in some cases), therefore increasing the need of a more self-responsible learning by the student. This means that the student has to work by himself (i.e. outside lectures and examples classes) on a regular basis. In practice this implies that the lecturer is supposed to plan the students work [8]. A number of powerful exercise generating systems have been proposed (see, e.g. [1, $6,10])$, and several e-learning systems have been developed (see, e.g. [3, 9, 2]). Also, a significant number of methodological studies in e-learning were done during the last years (see, e.g. $[8,7]$ ). Publications on these topics are surveyed in [4, 9].

We present a new intelligent interactive system called Passarola (name inspired by the well known Portuguese writer Saramago in his novel "Baltazar and Blimunda"). This system allows lecturers to describe exercises that combined together can be used to elaborate exam papers (including step-by-step solution and assessment). It can also be used to generate exercise sheets available online that can be used by students to self-regulate their study. The system surpasses the multiple choice questions assignments most e-learning environments are limited to, allowing the creation of more complex exercises in completely different domains (Music, Physics, Geography, etc.), using almost any kind of object (graphs, sound, source code, music score, etc.).

The use of dynamic programming allows the reuse of sub problems already programmed. It is a free open source system. In order to present this new system, we present a set of examples of exercises created for courses that range from Mathematics to Music and Geography. 
Exercises implemented in this system, are designed in a way that the student concentrates only on the essential. It can also provide a step by step solution and validation that helps the student to identify his potential mistakes. When properly used by lecturers it can prove to be a powerful tool for a student to autoregulate his study for a given course and indeed provide him the capability of learning to learn.

\section{The Passarola exercise generator}

Several robust exercise generating systems (most of them distributed as proprietary software) that use templates are currently available. These systems require a person with good computer skills, to implement exercises. Also, most popular systems allow true/false type exercises or multiple answers.

One of Passarola's design goal is to provide a simple, yet powerful, language that anyone with no computer science background, can use to describe exercises, that include a collection of heterogeneous objects, and allows the usage of more complex elements to provide answers, than simple boolean buttons, or input text areas.

The Passarola project was created to allow us to design exercises using simple reusable templates, predefined templates as often as possible, and simple and rich types. The use of rich notation and syntax (LaTeX based) for questions, solutions, and answers is also available in Passarola.

Passarola also allows the use and definition of transformation steps and calculations, the use of external calculators (functions and tools), and the creation of reusable functions and tools.

In order to do that a Domain Specific Language (DSL) Passarola was created for implementing and modeling: (1) rich exercises generation, (2) their step-bystep resolutions and explanations, and (3) evaluations, from simple to complex comparisons to specific algorithms or functions. The main characteristics of Passarola are:

- to provide random choices from static or dynamically calculated lists, allowing to control the complexity and constrain the domains to reasonable values;

- to provide coupled choices (selection of corresponding elements from two or more lists);

- to use LaTeX as the main authoring system to build documents (exercises and tests), allowing the reuse of huge amounts of styles and predefined common blocks;

- to use templating techniques to simplify LaTeX creation;

- to use previously computed elements as instances to describe more complex transformations and formulas, to simplify exercises, results, resolution steps, etc.;

- to use external languages and tools to perform specialized transfomation and calculations (e.g. Ma-xima, Perl, TikZ, and abcm2ps);

- the possibility of writing auxiliary functions in Maxima and Perl;

- it can be used locally or via web and it is distributed using an opensource license, growing with user contributions and needs.

A general idea of Passarola syntax is illustrated in the following example:

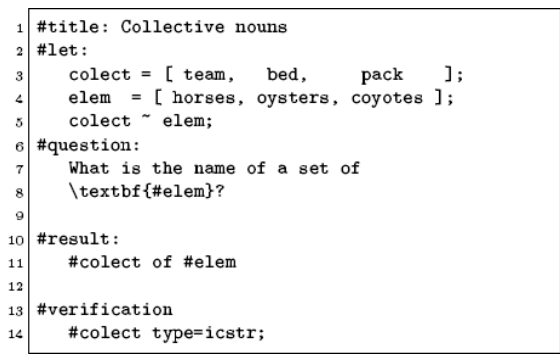

Fig. 1: Skeleton of an exercise in Passarola. 
Comments: line 3: select a random collect from the list; line 5: colect and elem are coupled elements; lines 7 and 8: the exercise text (in LaTeX) \#elem is replaced by the random chosen value; line 11: evaluation section; line 14: the validation function is a ignore-case string comparison of the submitted value with the value of collect.

\section{Case Studies}

In this section we present a few examples illustrating the features of Passarola in different domains of knowledge. A solution to an exercise can be represented as a sequence of "operators" (logical steps) $A_{k}$ applied to data "vectors" $x_{k}$ in order to deduce the next data vector $x_{k+1}=A_{k} x_{k}$. Schematically the solution can be represented as the following diagram:

$$
\begin{gathered}
x_{0} \rightarrow x_{1}=A_{1} x_{0} \rightarrow \cdots \rightarrow \\
x_{k+1=} A_{k+1} x_{k} \rightarrow \cdots \rightarrow x_{n}=A_{n} x_{n-1}
\end{gathered}
$$

If the operator $A_{k}$ is "invertible", then the vector $x_{k+1}$ can be reconstructed from the vector $x_{k}$. The exercise generation usually starts from an intermediate data vector $x_{k}$. Moving to the left we generate the exercise and moving to the right we obtain the answer:

$$
\begin{gathered}
x_{0} \leftarrow x_{1}=A_{1} x_{0} \leftarrow \cdots \leftarrow \\
x_{k+1=} A_{k+1} x_{k} \rightarrow \cdots \rightarrow x_{n}=A_{n} x_{n-1}
\end{gathered}
$$

The whole chain gives us the solution to the exercise.

\subsection{Mathematics: graph exercise}

Consider the following exercise: determine function $f(x)=\frac{P(x)}{Q(x)}, P(x)$ and $Q(x)$ being polynomials of degree two, subject to specified conditions. This function has the special form

$$
f(x)=\frac{1}{x-a}+\frac{1}{x-b}+c_{x}
$$

so that it has two vertical asymptotes, $x=a, x=b$, and a horizontal asymptote $y=c$. The parameters $a, b$, and $c$ are chosen to be integers. Additionally, two points are provided: the intersection point of $f$ with the $y$ axis, $A=(0, f(0))$, and with the horizontal asymptote, $B=\left(f^{-1}(c), c\right)$.

In Figure 2 it is presented an example of a possible function, where $A=(0,1 / 3)$ and $B=(2,1)$. Since in this case $a=1, b=3$ and $c=1$, the student should conclude that the function reads $f(x)=\frac{x^{2}-2 x-1}{x^{2}-4 x+a}$.

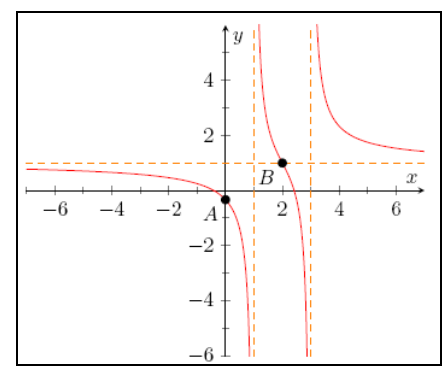

Fig. 2: Graph of the function $f$.

In order to generate this exercise together with its solution we follow the scheme:

$$
\text { exercise } \leftarrow a_{x} b_{x} c \rightarrow \text { solution, }
$$

meaning that just generating the parameters $a, b$, and $c$ allows, on one hand, to build up the exercise (the graph of $f$ and the points $A$ and $B$ ), and, on the other hand, to obtain the solution, which, in its general form, reads

$$
f(x)=\frac{c x^{2}+x(2-a c-b c)-a-b+a b c}{x^{2}-x(a+b)+a b} .
$$

The graph is obtained in Passarola using Tikz, a package for constructing graphics in LaTeX. The transformation of $f$ into one of its equivalent expressions (1) or (2) can be done with the help of Maxima using simplification instructions such as "ratsimp".

Passarola provides us the means to develop a correction system that gives the 
student a proper feedback. Let $f s$ denote the student's solution. The feedback to the student will follow the algorithm:

$$
\begin{aligned}
& \text { IF }(f s(x)=f(x)) \text { THEN } \\
& \text { "Function is ok" } \\
& \text { ELSE } \\
& \text { IF }(f s(a+\sigma) \rightarrow \infty) \text { THEN } \\
& \text { "Asymptote } x=a \text { is ok" } \\
& \text { IF }(f s(b+\delta) \rightarrow \infty) \text { THEN } \\
& \text { "Asymptote } x=b \text { is ok" } \\
& \text { IF (lim,- } f s(x)) \text { THEN } \\
& \text { "Asymptote y=c is ok" } \\
& \text { IF ( } \left.f s(0)=-\frac{2}{a}-\frac{2}{b}+c\right) \text { THEN } \\
& \text { "Point } A \text { is ok" } \\
& \text { IF ( } \left.f s\left(\frac{a+b}{2}\right)=c\right) \text { THEN } \\
& \text { "Point } B \text { is ok" }
\end{aligned}
$$

\subsection{Music: chord recognition}

In this section, we present and discuss an example of generating multi-media objects specified by a simple textual representation.

In order to generate music data (staves and audio) we use $\mathrm{ABC}$ music notation [5]. The sample of $A B C$ notation
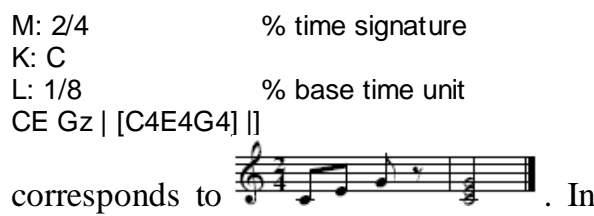
this way, we can define Passarola chord recognition exercise as given in Figure 3.

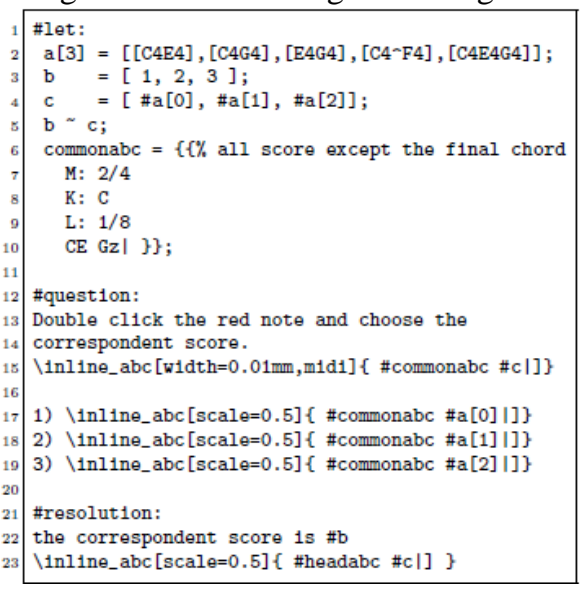

Fig. 3: Passarola code for the exercise.
With this, we generate the following exercise:

$$
\begin{aligned}
& \text { Double click in the red note and select the } \\
& \text { correspondent score }
\end{aligned}
$$

Fig. 4: Chord recognition exercise.

\subsection{Geography: rivers example}

Assume that the information we use to generate exercises is collected in various databases. For example, consider two relations: Rivers (name; length; tributaries; towns) and Towns (name; population; drainage basin). Instances of these relations for the longest river in Asia could be: Rivers (Yangtze; $6418 \mathrm{~km}$; Yalong, Min, Tuo, Jialing, Han, Wu, Yaun, Zi, Xiang, Gan, Huangpu; Yibin, Luzhou, Chongqing, ..., Shanghai, Jiangsu) and Towns (Hetchuan; 1.55 million; Jialing). Hechuan is a district in the northern part of Chongqing, China, located at the meeting point of the Jialing, $\mathrm{Fu}$, and $\mathrm{Qu}$ rivers. Hechuan is $54 \mathrm{~km}$ away from Chongqing city's central Yuzhong District. The sequence of logical steps is

Hetchuan $\leftarrow$ Jialing $\rightarrow$ Yangtze $\rightarrow$ Shangai.

From these relations one could formulate the question "Where does the precipitation fallen in Hetchuan region reach the Pacific Ocean?" The answer is: "In Shanghai-Jiangtsu", and the solution is: "Hetchuan belongs to the Jialing drainage basin. Jialing is a Yangtzes's tributary. Yangtze's base level is in ShanghaiJiangtsu."

\section{Conclusion}

In this work we presented an exercise generation system based on a DSL. Some decisions taken in the DSL design process were proved to be very important. Namely, Passarola provides: 
- division of a complex problem in several sub problems, reusing parts;

- possibility of embedding a language code (e.g. Perl and Maxima) inheriting a large amount of modules and functions, and providing a strong expressive power for user specific functions;

- a very powerful way of building a large variety of exercises via LaTeX-based templates. (LaTeX has a very rich set of packages that interfaces with a large set of external tools, such as gnuplot, graphviz, and $\mathrm{ABC}$ );

- table support and specific table importing notation to deal with tuples lines, providing a separation between processing and data, and constituting a mechanism for contributions of non-expert users (it is easy to add one more line to a table).

Acknowledgements: This research was financed by FEDER Funds through Programa Operacional Factores de Competitividade - COMPETE and by Portuguese Funds through FCT Fundação para a Ciência e a Tecnologia, within the Projects PEstC/MAT/UI0013/2011 and PEstOE/EEI/UI0752/2011.

\section{References}

[1] Belmonte, M. V. et al. "Automatic Generation of Problems in Webbased Tutors", Virtual Environments for Teaching \& Learning. World Scientific Publishing, Series on Innovative Intelligence 1, pp. 237-281, 2002.

[2] Cruz, P., Oliveira, P. \& Seabra, D. "Exercise Templates with Sage". Tbilisi Mathematical Journal (in press).

[3] Dettori, G., \& Persico, D. "Fostering Self-Regulated Learning through ICT". Institute for Education Tech- nology - National Research Council, Italy, 2011.

[4] Goguadze, G. "ActiveMath - Generation and Reuse of interactive exercises using domain reasoners and automated tutorial strategies". PhD Thesis, Universität des Saarlandes, 2011.

[5] Gonzato, G. "Making music with $\mathrm{ABC}$ plus", http://abcplus.sourceforge.net/\#ABC Guide, 2012.

[6] Gurtovoi, A., Guerman, A. \& Santos, C. "Sistema de Ensino Baseado no Computador: Gerador Automático dos Exercícios". Proc. of the Engenharia'2005 - Inovação e Desenvolvimento Conference. Covilhã, 21-23 November, 2005.

[7] Millán, E. et al. "Using Bayesian Networks to improve knowledge assessment" in Journal Computers and Education, Vol. 60, January, pp. 436447, 2013.

[8] Pereira, R. M. S., et al. "New elearning objects for the Mathematics courses from Engineering degrees: Design and Implementation of Question Banks in Maple T.A. using LaTeX". International Journal of Education and Information Technolo-gies, Vol. 4, pp. 7-14, 2010.

[9] Siegel, M. et al. "Promoting Teacher's Flexible Use of the Learning Sciences through case-based Problem Solving on the WWW: A Theoretical Design Approach", in Fourth International Conference of the Learning Sciences. NJ: Erlbaum, Mahwah, pp. 273-279, 2000.

[10] Tomás, A. P. \& Leal, J. P. “A CPLbased tool for Computer aided generation and solving of Maths exercises", Proc. of the Fifth International Symposium on Pratical Aspects of Declarative Languages, PADL'2003. Springer-Verlag, Lecture Notes in Computer Science 2562, pp. 223-240, 2003. 